\title{
Five-alpha Reductase Inhibitor Influences Expression of Androgen Receptor and HOXB13 in Human Hyperplastic Prostate Tissue
}

\author{
Chaeyong Jung, Youngwoong Park, Young-Rang Kim, Soo Bang Ryu, Taek Won Kang \\ Department of Urology and Department of Anatomy Research Institute of Medical Sciences, Chonnam \\ National University Medical School, Gwangju, Korea
}

\section{ABSTRACT}

Objectives: Five-alpha reductase inhibitors (5ARIs) are known as chemopreventive agents in prostate cancer with a risk of high-grade disease. This study evaluated the effects of 5ARI on androgen receptor (AR) and proteins involved in prostate cell growth such as HOXB13 expression in human prostate tissue and LNCaP prostate cancer cells.

Materials and Methods: We retrospectively selected 21 patients who underwent TURP between March 2007 and February 2010 for previously confirmed BPH by prostate biopsy. They were grouped into control (group $1, \mathrm{n}=9$ ) and 5ARI treatment (group 2, $\mathrm{n}=12$ ) before TURP. AR and HOXB13 expression in prostate tissue was evaluated by immunohistochemical staining. We tested the effect of 5ARI on the expression of AR, prostate specific antigen (PSA) and HOXB13 in LNCaP cells. Cells were assessed by Western blot analysis, MTT in vitro proliferation assay, and ELISA.

Results: Group 2 showed stronger reactivity for AR and HOXB13 than those of the group 1. MTT assay showed death of LNCaP cells at 25uM of 5ARI. At the same time, ELISA assay for PSA showed that 5ARI inhibited secretion of PSA in LNCaP cells. Western blot analysis showed that 5ARI did not greatly alter AR expression but it stimulated the expression of HOXB13.

Conclusions: These results demonstrated that 5ARI influences AR and HOXB13 expression in both LNCaP cells and human prostate tissue. In order to use 5ARI in chemoprevention of prostate cancer, we still need to clarify the influence of 5ARI in ARs and oncogenic proteins and its regulation pathway.

\section{ARTICLE INFO}

\section{Key words:}

5-alpha Reductase Inhibitors; Receptors, Androgen; H0XB13

Protein; Prostate

Int Braz J Urol. 2013; 39: 875-83

Submitted for publication:

February 01, 2013

Accepted after revision: August 01, 2013

\section{INTRODUCTION}

Five-alpha reductase inhibitor (5ARI) that is used to treat benign prostatic hyperplasia (BPH) blocks the conversion of testosterone (T) to dihydrotestosterone (DHT) and decreases intraprostatic DHT to as low as 30\% of normal value $(1,2)$. Testosterone and the more potent DHT are both primary androgens in the prostate, and are involved in the development of prostate cancer. During the past decades, 5ARIs have been investigated for their role in chemoprevention of prostate cancer (1-4).

Prostate cancer is an ideal candidate disease for chemoprevention because it is typically diagnosed in the elderly population with a relatively slower rate of growth and progression. Also, prostate growth and the development of prostate 
cancer are strongly influenced by androgens. In a large-scale population-based prevention study, the Prostate Cancer Prevention Trial (PCPT), finasteride had a $24.8 \%$ reduction effect on the prevalence of prostate cancer (4). However, a higher ratio of high-grade prostate cancer (6.4\% vs. 5.1\%) was found in men undergoing prostate cancer screening $(4,5)$. There are several hypotheses for explaining this phenomenon. A reduction in gland volume may permit a greater degree of ascertainment in men receiving finasteride, resulting in a proportionally higher rate of detection of high-grade cancers. And the reduced contribution of prostate-specific antigen (PSA) to BPH as finasteride makes changes in PSA more cancer specific (6-8). Li and Kim reported that molecular profiles on prostate carcinogenesis show that finasteride altered survival pathways and molecular adaptation associated with androgen receptor (AR) (9). Several studies have focused on the molecular effects of finasteride on prostate cancer development. A distinct biological involvement of chromosomal alterations and the AR gene were found in prostate cancer tissue that will change the expression profile of AR and oncogenes. Therefore, this study evaluated the effects of 5ARI on expression of AR and proteins involved in cell growth in human prostate tissue and cultured prostate cancer cells.

\section{MATERIALS AND METHODS}

A total of 21 patients submitted to an initial prostate biopsy and transurethral resection of the prostate (TURP) for BPH between March 2007 and February 2010 were reviewed, and 12 cases that had been treated with dutasteride $0.5 \mathrm{mg}$ daily for a minimum of 4 weeks before surgery were enrolled in the study in the 5ARI treatment group (group 2); nine patients who did not receive dutasteride treatment were selected as controls (group 1). All patients in the 5ARI treatment and control groups had confirmed BPH by an initial prostate biopsy. Transrectal ultrasonography (TRUS) guided prostate biopsy was performed in at least 8 cores or more of tissue targeting the peripheral zone at the apex, mid gland, and base on each side of the prostate. The tissue specimens obtained from biopsy and TURP were stained for histological examination and for immunohistochemical (IHC) assay. The research attained ethical approval from the institutional review board of Chonnam National University Hospital (IRB No. 06-070). The recommendations of the Declaration of Helsinki for biomedical research involving human subjects were followed.

Tissues were fixed in 10\% formalin for 24 $\mathrm{h}$, transferred to $70 \%$ ethanol, cleared in xylene and then embedded in paraffin. Sections $(5 \mu \mathrm{m})$ were cut and mounted on slides. Slides were hydrated through xylene and graded alcohol and equilibrated in PBS. Antigen retrieval was performed with sodium citrate $10 \mathrm{mM} \mathrm{pH} 6$, using a microwave for $10 \mathrm{~min}$ at $400 \mathrm{~W}$. Endogenous peroxidase activity was quenched with $3 \% \mathrm{H}_{2} \mathrm{O}_{2}$ in methanol. Non-specific binding was blocked with normal serum (Pierce, Rockford, IL). Polyclonal antibody against AR and HOXB13 was used for immunohistochemical staining of prostate gland sections at dilution of 1:200. All the slides were then washed several times in PBS and incubated with biotinylated goat anti-rabbit IgG (Amersham Biosciences Europe $\mathrm{GmbH}$, Milan, Italy) at dilution of 1:200, followed by peroxidase-labeled streptavidin (Amersham Biosciences Europe GmbH). The antigen-antibody complex was visualized by $10-\min$ incubation with diaminobenzidine tetrahydrochloride (Sigma Chemicals, St Louis, M0). Negative controls, made by excluding polyclonal antibodies from the reaction, showed no specific staining. Counterstaining was performed with hematoxylin (Sigma Chemicals, St Louis, M0) and cover slips were mounted on the slides with Eukitt (0. Kindler GmbH, Freiburg, Germany).

To assess immunoreactivity quantitatively, the mean percentage of positive staining of epithelial and stromal cells was determined by counting at least 10 random fields at both $40 \mathrm{x}$ and 400x magnification in each section. A positive immunoreaction was defined as a cell showing distinct nuclear AR staining. The IHC staining score was defined as the percentage of cells with positive staining. As a negative control, adjacent sections were immunostained without exposure to primary antibodies. The number of positive cells per high-power field was assessed as $0=$ no tumor 
cell staining, $1+=1-25 \%$ positively stained cells, $2+=26-50 \%$ of cells, and $3+=>50 \%$ of cells. Results were analyzed by a pathologist blinded to the clinical data. Two independent observers scored the percentage of positively stained cells per high-power field in the tissue sections.

LNCaP prostate cancer cells were grown in RPMI media containing 5\% charcoal/dextran-treated fetal bovine serum (CDT-FBS) for two days and then plated on 96-well plates and grown to $30 \%$ confluence. After this, in order to decide the optimal concentration of finasteride, LNCaP cells were grown under testosterone-deprived condition for 3 days. The next day, the cells were treated with 1,5 , and $25 \mu \mathrm{M}$ of finasteride and grown for up to five days. Media and/or with finasteride concentrations were changed every two days. Then, the cells were stained with $5 \mathrm{mg} / \mathrm{mL}$ MTT (3-[4,5-dimethylthiazol-2-yl]-2,5-diphenyltetrazolium bromide; thiazole blue, SIGMA, St. Louis, MO) solution and incubated for four hours at $37^{\circ}$ C. The reaction, in which mitochondrial dehydrogenase activity reduces the yellow MTT dye to a purple formazan, was stopped by adding DMSO (Dimethyl sulfoxide). The absorbance was measured at $570 \mathrm{~nm}$ using a microplate reader with SOFTmax PRO software (Molecular Devices, Sunnyvale, CA, USA).

LNCaP prostate cancer cells were plated in P100 culture dishes containing 5\% FBS-RPMI media. To deprive the cells of androgens, the cells were grown in RPMI media containing 5\% charcoal/dextran-treated fetal bovine serum (CDT-FBS) for 2 days. They were then treated with various concentrations of finasteride for 24 and $48 \mathrm{~h}$ and lysed in protein extraction buffer (1x TBS, 1\% NP-40, 0.5\% sodium deoxycholate), 0.1\% SDS and protease inhibitors. Twenty $\mu \mathrm{g}$ of total cell lysates were loaded onto 10\% Bis-Tris gel (Invitrogen) and separated using a Bio-rad electroporation system. After the proteins were transferred to a PVDF membrane, the primary antibodies were applied, followed by incubation with horse peroxidase-conjugated secondary antibodies. The following antibodies were used in this study. Polyclonal antibodies to HOXB13 have also been described previously (7). Antibodies to AR and $\beta$-actin were obtained from Santa Cruz Biotechnology Inc. The blots were developed using the ECL detection system (Pierce).

LNCaP prostate cancer cells were plated in P100 culture dishes containing 5\% FBS-RPMI media. To deprive the cells of androgens, the cells were grown in RPMI media containing 5\% CDT-FBS for 2 days. They were then treated with various concentrations of finasteride for 24 and $48 \mathrm{~h}$ and lysed in lysis buffer (25mM Tris $\bullet \mathrm{HCl}, 150 \mathrm{mM}$ $\mathrm{NaCl}, 1 \%$ NP-40, 1mM EDTA and 5\% glycerol). This assay employs the quantitative sandwich enzyme immunoassay technique (R\&D systems). Before loading samples, each lysate was quantified by Bradford assay and diluted with a buffered protein solution. The samples were added to each microplate well that had been precoated with a monoclonal antibody specific for kallikrein3/PSA and incubated at RT. After washing, an enzyme-linked polyclonal antibody specific for kallikrein3/PSA was added to the wells. Following a wash, a substrate solution was added to the wells and color develops in proportion to the amount of kallikrein3/PSA bound in the initial step. The color development was stopped and the intensity of the color was measured at $570 \mathrm{~nm}$ using a microplate reader with SOFTmax PRO software (Molecular Devices).

Statistical analyses were performed with SPSS $^{\circledast}$ software (version 13.0). Percentages were calculated for categorical variables. Chi-square test or Fisher's exact test was used to analyze categorical proportions. Multivariate analyses of AR immunoreactivity were conducted by fitted proportional odds polychotomous logistic regression model. P values less than 0.05 were considered significant.

\section{RESULTS}

The study included 21 patients who had confirmed BPH by prostate biopsy and were undergoing prostate surgery, of which 12 patients were given 5ARI before surgery and the remaining 9 patients served as controls. All patients were well matched for age, serum PSA, and transrectal ultrasound-estimated prostate volume, and all had a pathological diagnosis of BPH (Table-1). Sections from the hyperplastic prostate were stai- 
Table 1 - Patient characteristics.

\begin{tabular}{lccc}
\hline & Group 1 $(\mathrm{n}=9)$ & Group 2 $(\mathrm{n}=12)$ & p-value \\
\hline Age & $71.6(\mathrm{~s}=4.76)$ & $71.88(\mathrm{~s}=6.11)$ & 0.78 \\
Serum PSA $(\mathrm{ng} / \mathrm{dL})$ & $6.8(\mathrm{~s}=3.21)$ & $10.8(\mathrm{~s}=6.7)$ & 0.45 \\
Prostate volume $(\mathrm{mL})$ & $100.2(\mathrm{~s}=41.6)$ & $77.3(\mathrm{~s}=33.1)$ & 0.33 \\
\hline
\end{tabular}

PSA $=$ Prostate specific antigen

Group 1 = Control group

Group 2 = Experimental group

ned for expressions of AR and HOXB13 and the expression scores were statistically evaluated. Expressions of both AR and HOXB13 were confined to the nuclear compartments of prostatic luminal epithelial cells as shown in Figure-1. Expressions of both proteins were further evaluated by quantitative analysis as mentioned and demonstrated in Figure-2. As shown in Figure-2, AR expression was increased in group 2 (from 1.08 points to 1.57 points, $\mathrm{p}<0.05)$ and on the contrary AR expression was decreased in group 1 (from 1.75 points to 1.5 points, $\mathrm{p}<0.05)$. HOXB13 expression was increased in both the groups (from 1.67 points to 2.75 points in group 1 , from 0.71 points to 2.4 points in group $2, \mathrm{p}<0.05$ ) (Figure-2). These results suggest that even the mid-term result of 5ARI treatment showed upregulation of transcription factors which are considered to be positive growth regulators, namely AR and HOXB13.

To further demonstrate the effect of 5ARI on cultured prostate cells, LNCaP cells were employed. Currently, there are no hyperplastic prosta-

Figure 1 - Immunohistochemical analysis of AR and HOXB13 in human hyperplastic prostate tissue; A, B: androgen receptor, C, D: HOXB13

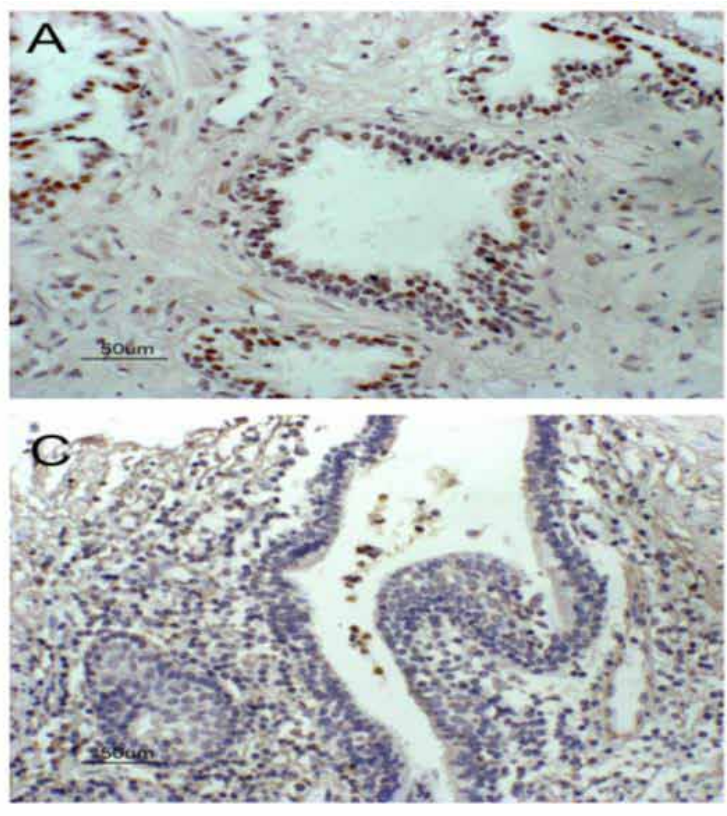

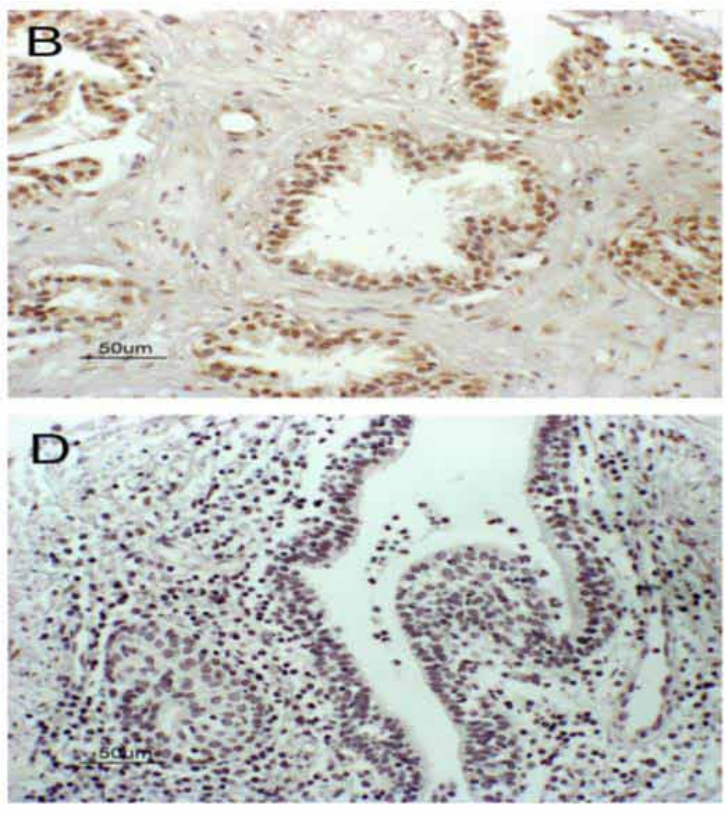


Figure 2 - Diagram showing AR and HOXB13 immunoreactivity in a hyperplastic prostate tissue section. (A: changes in AR expression, B: changes in HOXB13 expression)

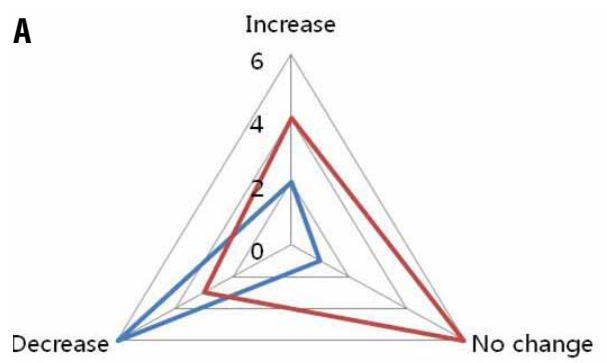

te cells or even normal prostate cells with intact AR expression. LNCaP cells are prostate cancer cells with a high androgen response. First, to decide the optimal concentration of finasteride, LN$\mathrm{CaP}$ cells were grown under testosterone-deprived condition for three days. Various doses of finasteride (final concentrations of $0,1,5,25 \mu \mathrm{M}$ ) were given to the cells every three days. Cell growth was measured by MTT in vitro proliferation assay every 2 days for up to 5 days. As shown in Figure-3, androgen-independent growth of LNCaP cells started to slow down at a dose of $5 \mu \mathrm{M}$ of finasteride, more distinctly at a dose of $25 \mu \mathrm{M}$ of finasteride. Further studies were carried out at a

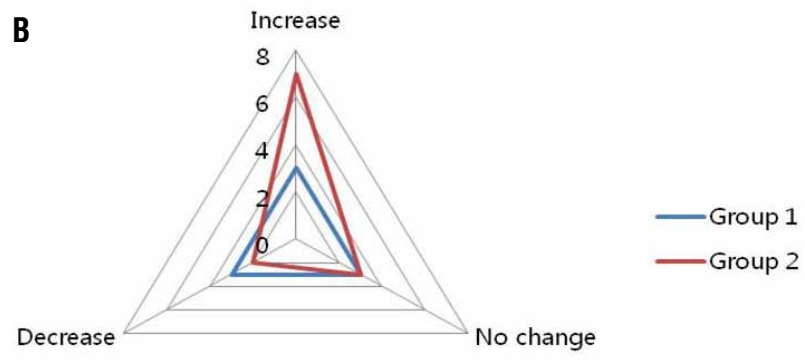

dose of $25 \mu \mathrm{M}$ of finasteride. At the same time, ELISA assay for PSA showed that 5ARI (at a dose of both 5 and $25 \mu \mathrm{M}$ ) inhibited secretion of PSA in LNCaP cells (Figure-4). Next, LNCaP cells were grown under similar conditions as mentioned above and $25 \mu \mathrm{M}$ of finasteride was given for up to 48 hours. In Western blot analysis, finasteride slightly down-regulated AR expression but it stimulated the expression of HOXB13 in whole lysates of 5ARI-treated cells (Figure-5).

These results suggest that HOXB13 expression was up-regulated by short-term finasteride treatment while AR expression was not very much affected by finasteride treatment.

Figure 3 - Finasteride inhibits growth of LNCaP cells. LNCaP cells were grown in RPMI media containing 5\% CDT-FBS for two days and cells were treated with 1, 5, and $25 \mu \mathrm{M}$ of finasteride and grown for up to five days. Then, the cells were stained with MTT solution.

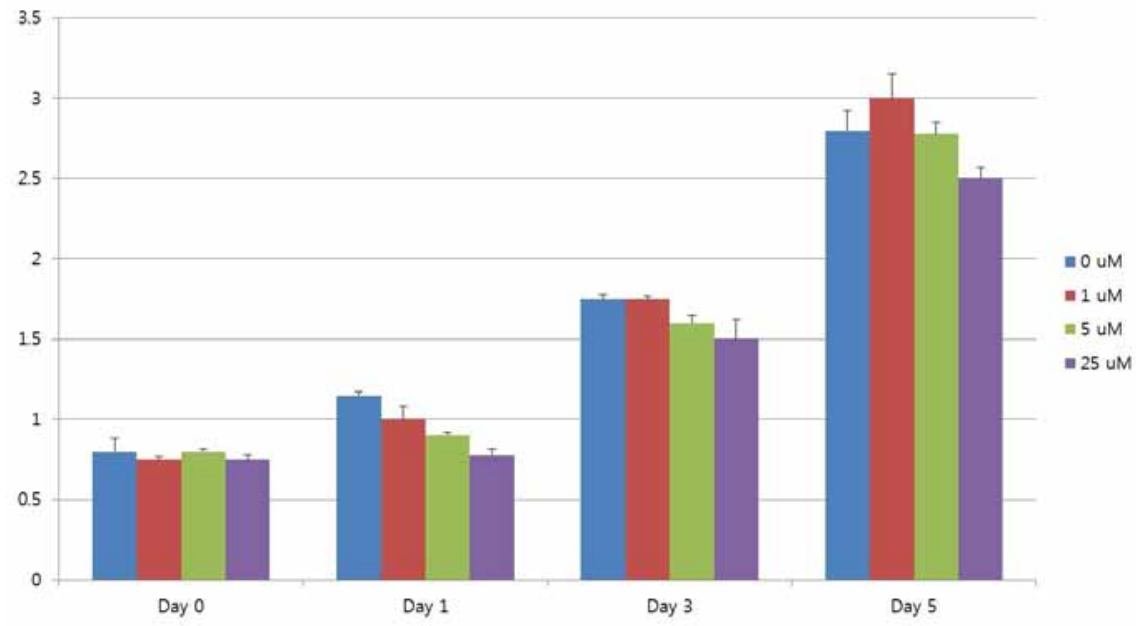


Figure 4 - Finasteride inhibits secretion of PSA in LNCaP cells: LNCaP cells were grown in RPMI media containing 5\% CDT-FBS for 2 days. They were then treated with various concentrations of finasteride for 24 and $\mathbf{4 8} \mathrm{h}$ and lysed. Quantikine human kallikrein3/PSA quantitative sandwich enzyme immunoassay technique (R\&D systems) was used to measure PSA levels.

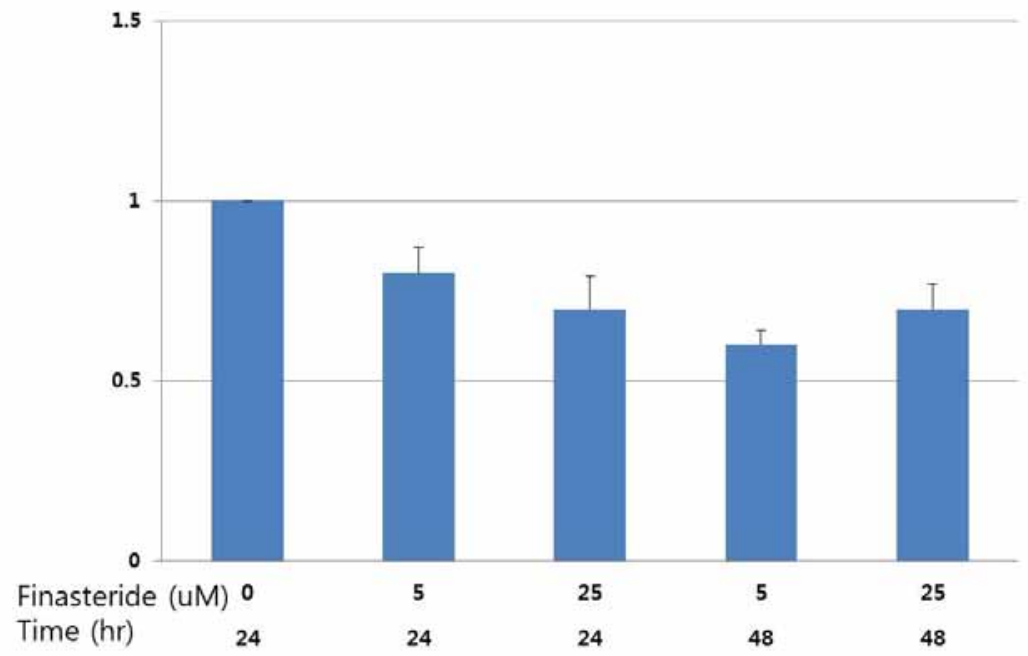

Figure 5 - Finasteride stimulates expression of HOXB13 but does not stimulate expression of AR. LNCaP cells were grown in $5 \%$ CDT-FBS-RPMI media. They were then treated with various concentrations of finasteride for 24 and $48 \mathrm{~h}$ and lysed and applied in Western blot analysis.

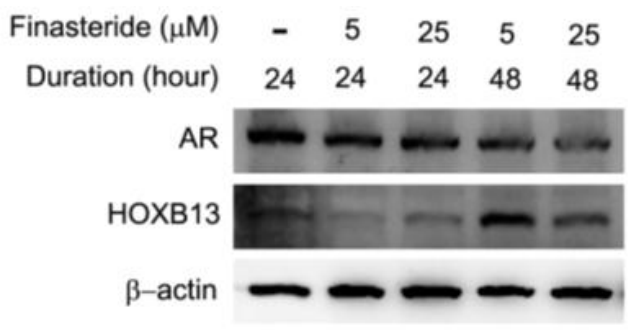

\section{DISCUSSION}

Inhibition of the synthesis of DHT for the chemoprevention is based on the understanding that androgen and AR are essential for pathogenesis of both benign prostatic epithelial cells and prostate cancer cells and that DHT is the most potent androgen that acts on the prostate $(10,11)$. Testosterone is converted to the more potent DHT by the enzymes 5AR type 1 and type 2 in the prostate. Both 5AR type 1 and type 2 are observed in
BPH. Currently, there are two types of 5ARIs available, finasteride inhibits 5AR type 2 and dutasteride inhibits both isoforms of 5AR. Dual inhibition by dutasteride has been shown to result in a greater degree of DHT suppression; however, there is no significant clinical difference between the two types of isoforms.

The PCPT study showed that finasteride significantly decreased the 7-year risk of prostate cancer by $24.8 \%$ versus placebo $(\mathrm{p}<0.001)$ in men with PSA 3.0ng/mL or less (4). However, high-grade tumors (Gleason score 7-10) were more common in the finasteride group than in the placebo group (37\% vs. $22.2 \%, p<0.001$ ). Some observers suggest that the likely cause of the bias could be that finasteride decreased PSA to a lesser extent in men with high-grade cancer, given that the sensitivity of PSA for detecting prostate cancer in the finasteride arm compared to the placebo arm was statistically significantly better (12). Another cause of the bias is likely due to the sampling density of a small gland volume and number of cores in the finasteride group, resulting in disproportionate sampling of the gland upon random needle biopsy as compared with the placebo group (1315). Recently, in the Reduction by Dutasteride of Prostate Cancer Events (REDUCE) trial, there was 
no significant increase in incidence of high-grade prostate cancer in the dutasteride arm, as compared with the placebo arm (1). There are some molecular biological hypotheses about this phenomenon. One reasonable explanation is that 5ARI alters the histological appearance of prostate cancers. It has been reported that androgen-deprived cancer has a significantly higher Gleason grade, lower nuclear grade, and smaller nucleolar diameter than untreated controls $(16,17)$. Another hypothesis is that finasteride induces the development of high-grade prostate cancer by changing the intraprostatic hormonal milieu (4). Finasteride can induce a low-DHT environment and this will provide the growth advantage for DHT-independent, high-grade prostate cancer clone (18).

The expression of $A R$ and 5AR are the main targets for the progression of prostate cancer. Roger et al. concluded that type 2 5AR expression decreased in prostate cancer, compared with benign prostate tissue and appears increased in high-grade than low-grade localized prostate cancer (19). The decrease in DHT with 5ARI was accompanied by a reciprocal increase in the serum and intraprostatic T levels. Due to the lower potency of T compared with DHT, 5ARI significantly decreased the total androgen effect (18). In this study, 5ARI significantly increased AR expression, matching the mechanism of hormone-refractory disease as $\mathrm{AR}$ overexpression or amplification. Also, HOXB13 expression was increased simultaneously. This means that 5ARI could influence the progression of undiagnosed prostate cancer.

For interpreting PCPT data on the molecular level, Bass et al. performed a study of patients receiving short-term, 30-day finasteride treatment before undergoing radical prostatectomy for localized prostate cancer (20). The expression of nuclear AR was not significantly different by immunohistochemistry quantification of benign and cancerous cells between the treatment and placebo groups. Hsieh et al. reported that the result of a long-term study was that more than 30 days of finasteride treatment alters the expression of AR in prostate epithelial cells, but this phenomenon cannot be seen in fewer than 30 days or longer than 180 days of treatment (21). In this study, IHC study revealed significant upregulation of ARs by
5ARI treatment. These short-term results are consistent with the results of the study by Bass et al., however, different results were obtained with the long-term use of 5ARI (20). This data showed interesting results, but there is a limitation due to the inter-individual differences in AR expression. In this study, the authors minimized the bias by using the prostate tissue from the same person before and after 5ARI use.

It is well established that Hox homeobox genes contain highly homologous homeodomains and are considered transcription factors that regulate axial regional specification during embryonic development. Hox genes are expressed in a tissue-specific and frequently stage-related fashion (22). The HOX 13 paralog is important to the development of accessory sexual organs, including the prostate. HOXB13 is required for normal differentiation and secretory function of the mouse prostate (23). In the previous studies, HOXB13 expression was correlated with the AR in both cultured prostate cancer cells and prostate xenograft models (24). In this in vitro study, we investigated the pattern of expression of HOXB13 in LNCaP prostate cancer cells after treatment with 5ARI.

Although 5ARI has encouraging data supporting its role in chemoprevention, the long-term effects of 5ARI in high-grade prostate cancer are still controversial. Although several reports have ascribed the phenomenon to bias in tissue sampling, uncertainty in the reproducibility of histological diagnosis, and increased PSA sensitivity, the main concern is the molecular profile of AR and the associated signaling pathway (25-27). In this study, 5ARI had been proven to be able to change the expression profile of AR and other oncogenic factors such as HOXB13. In the immunohistochemical study, we showed an increase in AR and HOXB13 expression in benign prostate tissue, and, we also showed an increase in the expression of HOXB13 in LNCaP prostate cancer cell model.

However, there are several limitations to this study. Enrolled patient's prostate tissues were retrospectively collected at a single center and so not grouped randomly and the sample size is relatively small. To clarify the long-term effect of 5ARI on prostate tissue, a longer period and quantified measurement methods are needed. Whether 
prostate cells adapt to the 5ARI effect or escape through a new growth pathway is yet to be determined. Consequently, we need more study to realize the long-term effects of 5ARI in the molecular profile.

\section{CONCLUSIONS}

Five alpha reductase inhibitor treatment influenced AR and HOXB13 expression in both LNCaP cells and human prostate tissue. In order to use 5ARI in chemoprevention of prostate cancer, we still need to clarify the influence of 5ARI in ARs and oncogenic proteins and its regulation pathway.

\section{ACKNOWLEDGEMENTS}

This study was financially supported by Chonnam National University and by a grant (CRI 120061-31) Chonnam National University Hospital Research Institute of Clinical Medicine.

\section{CONFLICT OF INTEREST}

\section{None declared.}

\section{REFERENCES}

1. Andriole GL, Bostwick DG, Brawley OW, Gomella LG, Marberger M, Montorsi F, et al.: Effect of dutasteride on the risk of prostate cancer. N Engl J Med. 2010; 362: 1192-202.

2. Gormley GJ, Stoner E, Bruskewitz RC, Imperato-McGinley J, Walsh PC, McConnell JD, Andriole GL, et al.: The effect of finasteride in men with benign prostatic hyperplasia. The Finasteride Study Group. N Engl J Med. 1992; 327: 1185-91.

3. D'Amico AV, Barry MJ: Prostate cancer prevention and finasteride. J Urol. 2006; 176: 2010-2; discussion 2012-3.

4. Thompson IM, Klein EA, Lippman SM, Coltman CA, Djavan B: Prevention of prostate cancer with finasteride: US/European perspective.Eur Urol. 2003; 44: 650-5.

5. Thompson IM, Goodman PJ, Tangen CM, Lucia MS, Miller GJ, Ford LG, et al.: The influence of finasteride on the development of prostate cancer. N Engl J Med. 2003; 349 215-24.

6. Cohen YC, Liu KS, Heyden NL, Carides AD, Anderson KM, Daifotis AG, et al.: Detection bias due to the effect of finasteride on prostate volume: a modeling approach for analysis of theProstate Cancer Prevention Trial. J Natl Cancer Inst. 2007; 99: 1366-74.
7. Redman MW, Tangen CM, Goodman PJ, Lucia MS, Coltman CA Jr, Thompson IM: Finasteride does not increase the risk of high-grade prostate cancer: a bias-adjusted modeling approach. Cancer Prev Res (Phila). 2008; 1: 174-81.

8. Kaplan SA, Roehrborn CG, Meehan AG, Liu KS, Carides $A D$, Binkowitz $B S$, et al.: PCPT: Evidence that finasteride reduces risk of most frequently detected intermediate- and high-grade(Gleason score 6 and 7) cancer.Urology. 2009; 73: 935-9.

9. Li J, Kim J: Molecular profiles of finasteride effects on prostate carcinogenesis. Cancer Prev Res (Phila). 2009; 2: 518-24.

10. Kim YR, Oh KJ, Park RY, Xuan NT, Kang TW, Kwon DD, et al.: HOXB13 promotes androgen independent growth of LNCaP prostate cancer cells by the activation of E2Fsignaling. Mol Cancer. 2010; 9: 124.

11. Koivisto PA, Schleutker J, Helin H, Ehren-van Eekelen C, Kallioniemi OP, Trapman J: Androgen receptor gene alterations and chromosomal gains and losses in prostate carcinomas appearingduring finasteride treatment for benign prostatic hyperplasia. Clin Cancer Res. 1999; 5: 3578-82.

12. Thompson IM, Chi C, Ankerst DP, Goodman PJ, Tangen CM, Lippman SM, Lucia MS, et al.: Effect of finasteride on the sensitivity of PSA for detecting prostate cancer. J Natl Cancer Inst. 2006; 98: 1128-33.

13. Kulkarni GS, Al-Azab R, Lockwood G, Toi A, Evans A, Trachtenberg J, et al.: Evidence for a biopsy derived grade artifact among larger prostate glands. J Urol. 2006; 175: 505-9.

14. Akduman B, Crawford ED: The PCPT: New findings, new insights, and clinical implications for the prevention of prostate cancer. Eur Urol Suppl. 2006; 5: 634-9.

15. Cohen YC, Liu KS, Heyden NL, Carides AD, Anderson KM, Daifotis AG, et al.: Detection bias due to the effect of finasteride on prostate volume: a modeling approach for analysis of theProstate Cancer Prevention Trial. J Natl Cancer Inst. 2007; 99: 1366-74.

16. Bostwick DG, Qian J, Civantos F, Roehrborn CG, Montironi $\mathrm{R}$ : Does finasteride alter the pathology of the prostate and cancer grading? Clin Prostate Cancer. 2004; 2: 228-35.

17. Bostwick DG, Ramnani D, Cheng L: Treatment changes in prostatic hyperplasia and cancer, including androgen deprivation therapy andradiotherapy. Urol Clin North Am. 1999; 26: 465-79.

18. Tindall DJ, Rittmaster RS: The rationale for inhibiting 5alpha-reductase isoenzymes in the prevention and treatment of prostatecancer. J Urol. 2008; 179: 1235-42. Erratum in: J Urol. 2008; 179: 2490.

19. Rittmaster R, Hahn RG, Ray P, Shannon JB, Wurzel R: Effect of dutasteride on intraprostatic androgen levels in men with benign prostatic hyperplasia or prostate cancer. Urology. 2008; 72: 808-12. 
20. Bass R, Perry B, Langenstroer P, Thrasher JB, Dennis KL, Tawfik 0, et al.: Effects of short-term finasteride on apoptotic factors and androgen receptors in prostate cancer cells. J Urol. 2009; 181: 615-9; discussion 619-20.

21. Hsieh JT, Chen SC, Yu HJ, Chang HC: Finasteride upregulates expression of androgen receptor in hyperplastic prostate and LNCaP cells: implications for chemoprevention of prostate cancer. Prostate. 2011; 71: 1115-21.

22. Kondo T, Zákány J, Innis JW, Duboule D: Of fingers, toes and penises. Nature. 1997; 390: 29.

23. Economides KD, Capecchi MR: Hoxb13 is required for normal differentiation and secretory function of the ventral prostate. Development. 2003; 130: 2061-9.

24. Jung C, Kim RS, Zhang HJ, Lee SJ, Jeng MH: HOXB13 induces growth suppression of prostate cancer cells as a repressor of hormone-activatedandrogen receptor signaling. Cancer Res. 2004; 64: 9185-92.

25. Klein EA, Thompson IM; Update on chemoprevention of prostate cancer. Curr Opin Urol. 2004; 14: 143-9.
26. Tindall DJ, Rittmaster RS: The rationale for inhibiting 5alpha-reductase isoenzymes in the prevention and treatment of prostatecancer. J Urol. 2008; 179: 1235-42. Erratum in: J Urol. 2008; 179: 2490.

27. Thomas LN, Douglas RC, Lazier CB, Too CK, Rittmaster RS, Tindall DJ: Type 1 and type 2 5alpha-reductase expression in the development and progression of prostate cancer. Eur Urol. 2008; 53: 244-52.

Correspondence address:

Taek Won Kang, MD

Department of Urology,

Chonnam National University Hospital and Medical School

8, Hak-dong, Dong-gu, Gwangju \#50

-757 , South Korea

Fax: + 8262 227-1643

E-mail: sydad@hanmail.net 